\title{
GRAPHICAL EVALUATION OF SPARSE DETERMINANTS
}

\author{
DANIEL DRUCKER AND DAVID M. GOLDSCHMIDT ${ }^{1}$
}

\begin{abstract}
In this paper, we show that the determinant of a matrix with entries in a commutative ring can be recursively computed by use of an associated directed graph whose circuits are assigned weights in the ring. This result provides an efficient means of calculating the determinants of sparse matrices. As an application, we compute the determinants of the Cartan matrices associated to the simple complex Lie algebras.
\end{abstract}

Let $M=\left(m_{i j}\right)$ be an $n \times n$ matrix with entries in a commutative ring $A$ with identity element 1 different from 0 . Define the associated graph of $M$ to be the directed graph $\Gamma$ with $n$ vertices (numbered from 1 to $n$ ) which has a directed edge $(i, j)$ from vertex $i$ to vertex $j$ precisely when $m_{i j} \neq 0(i=j$ is allowed). A circuit of length $k(1 \leqslant k \leqslant n)$ in $\Gamma$ is a $k$-cycle ${ }^{2} \gamma=\left[i_{1}, \ldots, i_{k}\right]$ such that $\left(i_{r}, i_{r+1}\right)$ (for $\left.1 \leqslant r<k\right)$ and $\left(i_{k}, i_{1}\right)$ are directed edges of $\Gamma$. The ring element $w(\gamma)=(-1)^{k-1} m_{i_{1}, i_{2}} \cdots m_{i_{k-1}, i_{k}} m_{i_{k}, i_{1}}\left(w(\gamma)=m_{i_{1} i_{1}}\right.$ if $\left.k=1\right)$ is called the weight of $\gamma$. We let $|M(\gamma)|$ denote the determinant of the submatrix $M(\gamma)$ obtained by deleting the rows and columns of $M$ numbered $i_{1}, \ldots, i_{k}$. When $k=n$, we set $|M(\gamma)|=1$.) For each vertex $i$, let $C(i)$ denote the set of all circuits to which $i$ belongs.

THEOREM. For any vertex $i,|M|=\Sigma_{\gamma \in C(i)} w(\gamma)|M(\gamma)|$.

Proof. Assume without loss of generality that $i=1$. The usual expansion of $|M|$ is

$$
|M|=\sum(\operatorname{sgn} \sigma) m_{1, \sigma(1)} m_{2, \sigma(2)} \cdots m_{n, \sigma(n)}
$$

where the sum ranges over all permutations $\sigma$ of $\{1,2, \ldots, n\}$. Without affecting the result, we can restrict the sum to those $\sigma$ for which

$$
m_{1, \sigma(1)} m_{\sigma(1), \sigma^{2}(1)} \cdots m_{\sigma^{k-1}(1), 1} \neq 0,
$$

where $k$ is the smallest positive integer such that $\sigma^{k}(1)=1$. In terms of the associated graph $\Gamma$, this restricts the sum to the permutations $\sigma$ for which the $k$-cycle $\left[1, \sigma(1), \ldots, \sigma^{k-1}(1)\right]$ is a circuit of $\Gamma$ with nonzero weight. Summing over all $\sigma$ corresponding to a single circuit $\gamma$ with $w(\gamma) \neq 0$ yields the partial

Presented to the Society, March 31, 1979; received by the editors May 8, 1978 and, in revised form, December 30, 1978.

AMS (MOS) subject classifications (1970). Primary 15A15, 05C20; Secondary 17B20.

Key words and phrases. Determinant, sparse matrix, directed graph, circuit, Cartan matrix, Dynkin diagram.

${ }^{1}$ Partially supported by NSF MCS 77-03719.

${ }^{2}$ By the $k$-cycle $\left[i_{1}, \ldots, i_{k}\right]$ we mean the cyclic permutation of the sequence of distinct indices $\left(i_{1}, \ldots, i_{k}\right)$. 
sum $w(\gamma)|M(\gamma)|$. If we then sum over all circuits with nonzero weight in $C(1)$, we obtain the desired result.

If $\gamma=\left[i_{1}, \ldots, i_{k}\right]$ is a circuit of $\Gamma$, then the associated graph of $M(\gamma)$ is the subgraph $\Gamma(\gamma)$ obtained from $\Gamma$ by deleting the vertices $i_{1}, \ldots, i_{k}$ and all directed edges to which they belong. Every circuit of $\Gamma(\gamma)$ is a circuit of $\Gamma$, so it is clear that repeated applications of the theorem enable us to express $|M|$ solely in terms of weights of circuits in $\Gamma$. This means that we can calculate $|M|$ just by looking at the graph $\Gamma$ with its edges labelled by the appropriate ring elements $m_{i j}$. If we let $|\Gamma|$ denote the determinant of the matrix over $A$ with associated labelled graph $\Gamma$, then the conclusion of the theorem is that

$$
|\Gamma|=\sum_{\gamma \in C(i)} w(\gamma)|\Gamma(\gamma)| \text { for each vertex } i \text { in } \Gamma \text {. }
$$

It is helpful to notice that a labelled graph $\Gamma$ with connected components $\Gamma_{1}, \Gamma_{2}, \ldots, \Gamma_{t}$ has determinant

$$
|\Gamma|=\left|\Gamma_{1}\right| \cdot\left|\Gamma_{2}\right| \cdots\left|\Gamma_{t}\right|
$$

(The vertices of $\Gamma$ can be renumbered so that $\Gamma$ is associated to a matrix $M$ whose nonzero entries all belong to square submatrices $M_{1}, \ldots, M_{t}$ along the diagonal. These matrices are associated to $\Gamma_{1}, \ldots, \Gamma_{t}$, so $|\Gamma|=|M|=\left|M_{1}\right|$ $\left.\cdots \cdot\left|M_{t}\right|=\left|\Gamma_{1}\right| \cdots \cdot\left|\Gamma_{t}\right| \cdot\right)$

To simplify our graphs, we make some conventions. Rather than represent circuits of length 1 by directed edges of the form $(i, i)$, we write $m_{i i}$ next to the vertex $i$, even when $m_{i i}=0$. When necessary, we also label each vertex $i$ with the underlined number $\underline{i}$.

EXAMPLE 1. The associated labelled graph of a matrix $M$ automatically displays any easy-to-overlook simplifications in the calculation of $|\boldsymbol{M}|$ that would result from interchanges of rows and/or columns in $M$. For example, the matrix

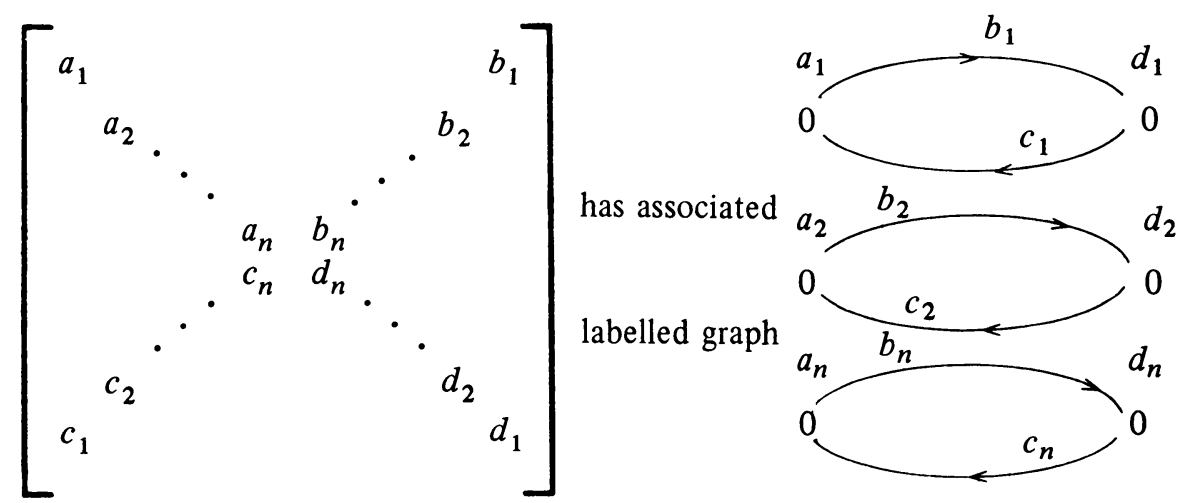

so its determinant is immediately seen to be

$$
\prod_{i=1}^{n}\left(a_{i} d_{i}-b_{i} c_{i}\right), \quad \text { i.e., } \quad \prod_{i=1}^{n}\left|\begin{array}{cc}
a_{i} & b_{i} \\
c_{i} & d_{i}
\end{array}\right| \text {. }
$$


EXAMPLE 2 (cf. [3, pp. 251-252]). Let $M$ be the companion matrix of a monic polynomial $p=x^{n}+c_{n-1} x^{n-1}+\cdots+c_{1} x+c_{0}$ with coefficients in a field $F$. If $I$ is the $n \times n$ identity matrix, then

$$
x I-M=\left[\begin{array}{rrrrrl}
x & 0 & 0 & \cdots & 0 & c_{0} \\
-1 & x & 0 & \cdots & 0 & c_{1} \\
0 & -1 & x & \cdots & 0 & c_{2} \\
\vdots & \vdots & \vdots & & \vdots & \vdots \\
\vdots & \vdots & \vdots & & \vdots & \vdots \\
0 & 0 & 0 & \cdots & x & c_{n-2} \\
0 & 0 & 0 & \cdots & -1 & x+c_{n-1}
\end{array}\right] .
$$

We will show that the characteristic polynomial of $M$ is $p$.

The associated graph $\Gamma$ of $x I-M$ is

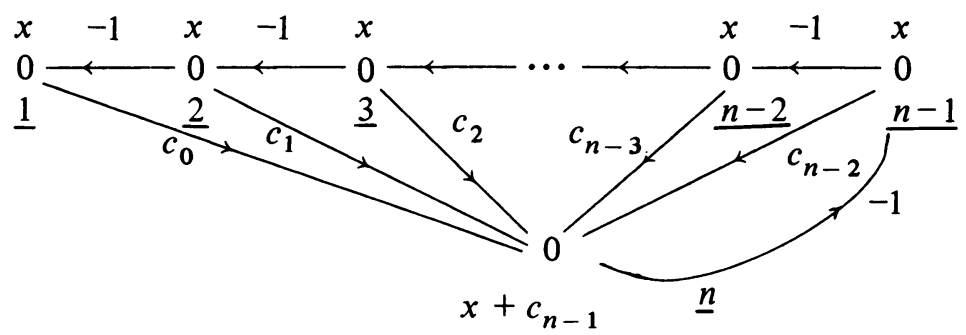

Its circuits through vertex $n$ are $\gamma_{i}=[n, n-1, \ldots, i], 1<i<n$. The corresponding weights are $w\left(\gamma_{n}\right)=x+c_{n-1}$ and

$$
w\left(\gamma_{i}\right)=(-1)^{n-i} m_{n, n-1} \cdots m_{i+1, i} m_{i, n}=c_{i-1}
$$

for $1<i<n-1$. Also

$$
\left|\Gamma\left(\gamma_{i}\right)\right|=\left|\begin{array}{l}
x \\
0 \longleftarrow \cdots \longleftarrow \begin{array}{c}
x \\
1 \\
1
\end{array} \mid \underline{i-1}
\end{array}\right|=x^{i-1}
$$

for all $i$ (by repeated application of (1)-note that $\Gamma\left(\gamma_{i}\right)$ has no circuits of length $>1$ ). Applying (1) to vertex $n$ yields

$$
|x I-M|=\left(x+c_{n-1}\right) x^{n-1}+\sum_{i=1}^{n-1} c_{i-1} x^{i-1}=p,
$$

as desired.

EXAMPLE 3 (Determinants of Cartan matrices). To each simple complex Lie 
algebra is associated a Cartan matrix $M=\left(m_{\alpha \beta}\right)$ whose entries are rational integers. The associated directed labelled graph is closely related to the so-called Dynkin diagram (see [1], [3], [4], [5]) and one easily verifies that these graphs are as follows:

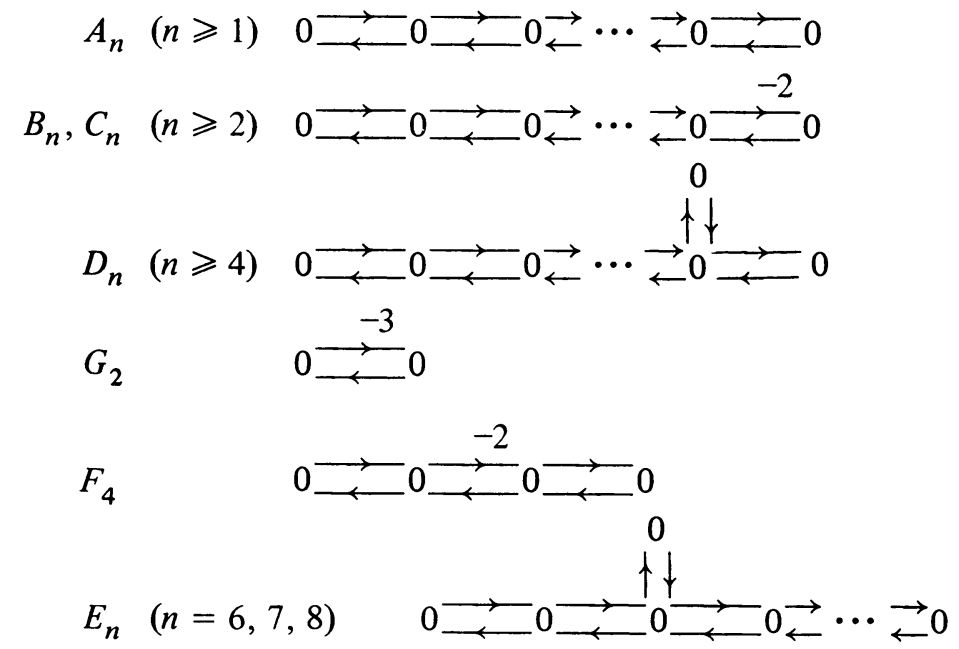

where it is understood that each vertex should be labelled 2 and that each unlabelled edge should be labelled -1 . Note that there are no directed circuits of length $>2$. If we denote by $a_{n}, b c_{n}, d_{n}, e_{n}, f_{4}, g_{2}$ the values of the corresponding determinants, formula (1) yields

$$
a_{n}=2 a_{n-1}-a_{n-2} \text {, }
$$

hence

$$
a_{n}-a_{n-1}=a_{n-1}-a_{n-2}=\cdots=a_{2}-a_{1}=1 \text {, }
$$

and we get

$$
a_{n}=n+1
$$

For $B_{n}$ and $C_{n}$ we get

$$
b c_{n}=2 a_{n-1}-2 a_{n-2}=2 n-2(n-1)=2 .
$$

For $D_{n}$,

$$
d_{n}=2 a_{n-1}-a_{n-3} \cdot a_{1}=2 n-2(n-2)=4 .
$$

The remaining values are easily calculated to be

$$
f_{4}=g_{2}=1
$$

and

$$
e_{n}=2 a_{n-1}-a_{2} \cdot a_{n-4}=2 n-3(n-3)=9-n .
$$

\section{REFERENCES}

1. N. Bourbaki, Éléments de mathematique, Fasc. 34, Groupes et algèbres de Lie, Chapitres IV, V, VI, Actualités Sci. Indust., no. 1337 Hermann, Paris, 1968. 
2. K. Hoffman and R. Kunze, Linear algebra, 2nd ed., Prentice-Hall, Englewood Cliffs, N. J., 1971.

3. J. E. Humphreys, Introduction to Lie algebras and representation theory,.Springer-Verlag, Berlin and New York, 1972.

4. N. Jacobson, Lie algebras, Interscience, New York, 1962.

5. J.-P. Serre, Algèbres de Lie semi-simples complexes, Benjamin, New York, 1966.

Department of Mathematics, Wayne State University, Detrort, Michigan 48202

Department of Mathematics, University of California, Berkeley, California 94720 